\title{
Balkanologie
}

Balkanologie Revue d'études pluridisciplinaires

Vol. XI, $n^{\circ}$ 1-2 | 2008

Volume XI Numéro 1-2

\section{Dynamique des réseaux sociaux dans un contexte de migrations intensives : le cas de Satovča (Rhodopes bulgares)}

\section{Margarita Karamihova}

Traducteur : Bernard Lory

\section{(2) OpenEdition}

\section{Journals}

Édition électronique

URL : http://journals.openedition.org/balkanologie/1202

DOI : 10.4000/balkanologie.1202

ISSN : 1965-0582

\section{Éditeur}

Association française d'études sur les Balkans (Afebalk)

\section{Référence électronique}

Margarita Karamihova, « Dynamique des réseaux sociaux dans un contexte de migrations intensives : le cas de Satovča (Rhodopes bulgares) », Balkanologie [En ligne], Vol. XI, n 1-2 | 2008, mis en ligne le

31 décembre 2008, consulté le 17 décembre 2020. URL : http://journals.openedition.org/balkanologie/ 1202 ; DOI : https://doi.org/10.4000/balkanologie.1202

Ce document a été généré automatiquement le 17 décembre 2020.

(c) Tous droits réservés 


\title{
Dynamique des réseaux sociaux dans un contexte de migrations intensives : le cas de Satovča (Rhodopes bulgares)
}

\author{
Margarita Karamihova \\ Traduction : Bernard Lory
}

Cet article présente une analyse de la dynamique des différents réseaux sociaux dans un village montagnard et frontalier de Bulgarie, soumis à des courants migratoires intensifs. La notion de réseaux sociaux permet de décrire et d'analyser dans leur évolution les interactions réciproques d'acteurs impliqués dans des noyaux sociaux complexes plus ou moins durables. La description de ces processus complexes, produits d'un milieu culturel qui n'est que partiellement connu pour ses phases antérieures, constitue un sérieux défi scientifique, pour lequel il faut mobiliser toutes les ressources propres à l'ethnologie ou empruntées aux sciences humanitaires voisines. Le postulat de départ de la description du processus en cours est classique : chaque individu entre en interaction complexe avec des personnes qui appartiennent à des groupes de diverses natures. Ces interactions se fondent sur des rapports de caractères variables, qui tissent des réseaux intriqués. Une des possibilités consiste à classifier ces rapports dans la durée : liens de courte durée (par exemple vendeur-acheteur) ou liens de longue durée (par exemple liens d'amitié, de parenté). Cette première classification conduit à la suivante, celle qui juge de la force et de l'intensité des rapports. Il est indéniable qu'une caractéristique générale de tous ces rapports est leur tendance à se modifier continuellement. Selon leur importance dans la stratégie individuelle de chacun, à chaque moment donné, des liens de courte durée peuvent manifester une tendance à se transformer en liens de longue durée, et réciproquement. Il est plus pertinent pour notre analyse de considérer les rapports en fonction de leur force (White, Houseman 2), c'est à dire de leur importance ou leur intensité. Ainsi les rapports par lesquels chaque individu élabore ses réseaux seront-ils qualifiés de faibles (en général de courte durée) ou de forts (de longue durée). Les rapports faibles se caractérisent par un 
investissement limité en temps et en ressources (White, Houseman 2). Les rapports forts exigent un investissement plus grand (souvent répété) dans le temps ainsi qu'un engagement émotionnel. Pour parvenir à positionner un individu sur la carte sociale des réseaux, il importe de déterminer combien de liens et de quelle nature il gère et maintient en activité. Les études des sociétés urbaines d'Amérique du Nord contemporaines montrent que les rapports forts (de type durable) constituent entre 35 et $40 \%$ du réseau d'un individu donné (Wetherell, Plakans, Wellman, 1994: 648). Jusqu'à présent on n'a pas procédé à des études quantitatives dans ce domaine en Bulgarie. Par ailleurs, la communauté qui fait l'objet de cette présentation présente des paramètres fort différents, ainsi qu'il sera montré. Néanmoins ce pourcentage nous donne un certain ordre de grandeur.

2 Les rapports faibles et forts qui tissent les différents réseaux tendront à se modifier au cours de l'étude de ce milieu, resté longtemps quasi-statique, puis connaissant une très forte tendance à l'émigration, due à l'action de facteurs nouveaux. Durant l'observation ici présentée émergera un jeu complexe et mouvant, modifiant la force et l'importance des rapports tissant les différents réseaux sociaux, impliquant des noyaux en mouvement, aux lieux et aux frontières variables, et ce dans un laps de temps très court. La véracité de l'axiome qui veut que l'essence d'une communauté réside dans sa structure sociale, et non pas dans sa structure spatiale, s'y vérifie pleinement (Wetherell, Plakans, Wellman, $1994: 645$ ). On peut s'attendre à ce que ce jeu produise bientôt de nouveaux éléments culturels et que se dessinent de nouvelles tendances modifiant l'importance et la configuration spatiale des noyaux résiliaires (pour l'individu et le sous-groupe auquel il participe immédiatement). L'objet de cet article s'inscrit dans le cadre d'un processus intense, enclenché depuis une dizaine d'années, c'est à dire sur un laps de temps suffisant pour que les phénomènes et les tendances acquièrent assez de lisibilité.

\section{Cadre général}

3 Au début de la transition démocratique en Bulgarie, en même temps que les autres pays $\mathrm{du}$ bloc socialiste (1989), s'enclenchent des processus migratoires rapides et très intenses. Ils s'opèrent aussi bien à l'intérieur que vers l'extérieur du pays. Pour la seule période 1990-1992, plus de 250000 personnes ont officiellement quitté le pays. La Bulgarie et les Balkans ont connu des migrations volontaires ou forcées tout au long du $\mathrm{XX}^{\mathrm{e}}$ siècle. Les exodes en provenance de certains pays voisins, résultat des nombreuses guerres et conflits politiques, ont laissé un souvenir traumatisant, toujours vivant, dans les mémoires familiales et font partie de l'expérience individuelle de bon nombre de citoyens bulgares. A l'époque du totalitarisme (1944-1989) la nation socialiste était enclose dans les frontières étatiques, et ses mouvements migratoires, étroitement encadrés, concernaient des groupes restreints selon un axe : village - petite ville grande ville. Un pourcentage infime de la population pouvait effectuer des migrations de travail temporaire dans le cadre du Conseil d'Assistance Economique Mutuelle (CAEM / Comecon) ou vers le monde arabe, sous le contrôle étroit de l'Etat. Des cas individuels parvenaient à franchir le Rideau de Fer et à s'installer en Europe occidentale, aux USA ou en Australie. On aurait pu croire que l'habitude de voyager de par le monde s'était perdue. 
4 Après 1989, les citoyens bulgares adoptent un comportement migratoire comparable à celui des autres pays de l'ex-Bloc de l'Est (que l'on désigne à présent par euphémisme comme « pays en transition économique»). De 1990 à 1994, entre 1.500000 et 1.900000 personnes ont émigré de ces pays (International... $2002: 25)$. Ces "grandes invasions barbares " d'un nouveau genre n'ont guère surpris les spécialistes. La région a une longue tradition de vagues migratoires massives et un potentiel migratoire important (International... $2002: 43$ ).

5 La longue et difficile transition vers l'économie de marché, commencée en 1989 sous une économie dirigée fortement érodée, a touché particulièrement durement les régions montagneuses et frontalières de Bulgarie. Les ateliers locaux des usines ont été fermés et l'agriculture décollectivisée. Cette forme de transition est qualifiée de violence économique par certains chercheurs (Pickles 2002: 6). En situation de violence économique, les groupes et les régions marginales sont toujours sacrifiés (Creed 1998). C'est également le cas de la région frontalière du massif des Rhodopes qui nous intéresse ici. La nature de la plupart des entreprises implantées dans les Rhodopes conditionnait aussi leur impuissance à évoluer durant la période de transition : elles avaient été créées comme filiales de conglomérats industriels et n'avaient pas suffisamment d'autonomie pour assurer une transition moins heurtée, aussi furentelles les première victimes de la déstructuration massive (Pickles 2002:6). La perte de leurs anciens marchés ou d'une grande partie de leur production (par exemple le tabac) n'a fait que précipiter la tragédie économique pour la population montagnarde. Selon un rapport officiel du Ministère de l'Aménagement et du Développement Régional, les départements de Smoljan et de Haskovo, qui couvrent les Rhodopes centraux et orientaux, sont en 1996 parmi les quatre départements les plus affectés par la crise (les deux autres, ceux de Pernik et de Vidin sont situés sur la frontière occidentale). La situation ne s'est pas arrangée durant les années suivantes. La violence économique est qualifiée de " génocide économique » dans le village rhodopéen que nous avons étudié ; elle frappe à égalité la population montagnarde de tous les groupes ethno-culturels (Bulgares chrétiens, Bulgares musulmans, Turcs, Roms).

\section{Cadre idéologique}

6 Ces seize dernières années, les ethnologues et historiens de Bulgarie et du reste du monde se sont à maintes reprises penchés sur la culture et le sort historique des Bulgares musulmans (Todorova, Marushiakova \& Popov, Konstantinov, Karagianis, etc.) Ce groupe important, qui se compose d'une multitude de sous-groupes, parle la langue bulgare et confesse l'Islam. Les données du recensement de 2001 permettent, avec quelques réserves, de les évaluer à 83000 personnes, soit 1,2 \% de la population totale (Marushiakova \& Popov). Dans la littérature scientifique ce groupe est également désigné comme Pomaks, terme qui a une connotation extrêmement offensante pour lui. Dès la fin du XIX siècle, alors que se constituait l'idéologie nationale bulgare, les Bulgares musulmans furent stigmatisés comme des «Bulgares apostats». Ceci détermina une politique de longue haleine visant à leur assimilation. Sous le Troisième Royaume bulgare (1878-1944) et sous le régime totalitaire (1944-1989), cette politique se manifesta crûment dans plusieurs opérations de baptêmes forcés, conçues comme des opérations de bulgarisation: en 1912-1913, à la fin des années 1930 et au début des années 1940, au début des années 1960, au début des années 1970 (Gruev, Karamihova, 
Marushiakova \& Popov). Cette politique d'assimilation mettait en jeu toute la gamme clairement répertoriée par O'Leary (O'Leary, $1993: 1-40)$ : hégémonie et contrôle total de la part de la majorité, déportations à l'intérieur du pays, émigration forcée, tentatives d'intégration sur une base supra-ethnique ( la fraternité de la classe ouvrière socialiste »), assimilation forcée. Lors de chaque offensive les noms musulmans furent remplacés par des noms "bulgares ". Après la dernière opération achevée vers 1974, le pouvoir totalitaire estimait avoir définitivement assimilé les Bulgares musulmans (Marushiakova \& Popov), mais jusqu'en 1989 il ne cessait de contrôler étroitement leur vie publique et privée, afin de ne laisser passer la moindre pratique qui puisse être perçue comme une »spécificité pomaque». Au début des années 1990, les sociologues purent établir qu'à peine $5 \%$ de chaque groupe confessionnel se percevait et s'auto-identifiait sur le plan religieux comme des pratiquants réguliers. De fait, l'idée que « la religion est l'opium des peuples » avait été inculquée dans la conscience de masse; dans un monde moderne et rationnel, elle ne pouvait donc subsister, vouée à un lent dépérissement, que dans les couches de population les plus pauvres et les moins éduquées, c'est à dire, pensait-on, parmi les personnes âgées et dans les campagnes.

\section{Le village de Satovča, la commune de Satovča}

7 Le village de Satovča a une population de 2400 habitants. C'est le centre administratif d'une commune, dont la limite méridionale coïncide avec la frontière entre la Bulgarie et la Grèce. Le village se situe à 1029 mètres d'altitude.

8 Selon la statistique officielle, y résident des Bulgares musulmans (50\%), des Bulgares orthodoxes (40\%) et des Roms musulmans, qui depuis peu passent rapidement et massivement à l'évangélisme (10\%). Le village possède une église orthodoxe et une mosquée, toutes deux en fonction. Les différents groupes vivent depuis longtemps dans des quartiers mélangés. Les Bulgares musulmans constituent un groupe significatif dans le village et dans la commune. Suite à la politique d'athéisme agressif du régime totalitaire, renforçant la tendance générale à la sécularisation qui caractérise les temps modernes, les habitants du village et de la commune faisaient preuve, en 2002, d'un degré de religiosité très faible. Les signes extérieurs de l'identité religieuse musulmane dans l'espace publique se réduisaient au seul minaret de la mosquée. Les générations nées après 1944, qui ont bénéficié d'une éducation toujours plus poussée en dehors du cadre montagnard, ont délaissé les connaissances religieuses et rituelles propres à leur groupe, les jugeant inutiles pour leurs stratégies individuelles. L'érosion des connaissances religieuses, y compris parmi les responsables spirituels, l'absence de contrôle de la stricte exécution des prescriptions rituelles (et même, au contraire, l'obligation de les oublier), le nouveau rythme et les nouvelles règles de la vie moderne, tout cela a conduit à une forte sécularisation des consciences chez eux, comme d'ailleurs pour l'ensemble des citoyens bulgares.

9 Le système, lié à la société pré-industrielle, que l'on désigne traditionnellement comme la culture populaire s'est irréversiblement décomposé au début des années $60 \mathrm{du} \mathrm{XX}^{\mathrm{e}}$ siècle. C'est en 1961 que la dernière noce traditionnelle a été célébrée à Satovča. Vers la même époque la pratique du jeûne du ramadan est tombée en désuétude. La religion semblait donc en voie d'extinction; néanmoins l'appartenance à un groupe confessionnel gardait une forte valeur de signe d'appartenance ou de différenciation. 
10 La politique systématique d'assimilation a créé une incertitude et la peur d'une nouvelle «bulgarisation» qui sont devenues une composante permanente de la vie quotidienne. Le complexe pomak continue de peser de nos jours sur les membres de ce groupe. Depuis les dernières années, les Bulgares musulmans des Rhodopes ont l'espoir qu'avec l'entrée de la Bulgarie dans l'Union Européenne, leur problème identitaire disparaîtra enfin. Ils se fondent en cela sur l'expérience des Pomaks de Grèce (Mihail, 2003), avec lesquels nombre d'entre eux entretiennent des liens de parenté et de voisinage vivaces, malgré les limitations imposées par le régime frontalier.

11 L'activité des habitants du village de Satovča après 1989 se réduit à trois petits ateliers de confection, une agriculture de montagne parcellisée (culture du tabac et élevage de bovins), le commerce local, l'administration communale, l'exploitation forestière, l'école (cursus primaire et secondaire). La culture et le conditionnement primaire du tabac a été une des caractéristiques de l'économie locale presque tout au long du XX siècle. Mais l'absence de possibilité d'investir l'argent gagné par cette activité pénible et laborieuse, quoique relativement bien rétribuée à l'époque socialiste, est à l'origine de l'usage de construire de grandes maisons à plusieurs étages. La natalité parmi les musulmans restée relativement élevée à l'échelle du pays (en moyenne 3 enfants par famille, contre 1,5 pour l'ensemble de la population), les liens familiaux et de parenté solides, la solidarité interne au groupe et les contraintes du terrain montagneux ont contribué à l'adoption du principe : un étage par fils. Malgré l'incertitude permanente née de l'interminable processus de privatisation du secteur du tabac, cette culture reste aujourd'hui encore une source de revenus complémentaires pour les ménages de Satovča. Les revenus du tabac permettent de maintenir un niveau de vie à peu près correct ou bien sont investis dans les études des enfants. La culture du tabac constitue l'unique revenu annuel en argent pour une très petite proportion des habitants du village seulement.

Selon les données officielles, le taux de chômage du village est relativement faible. Mais les employés de la commune avec qui j'ai eu l'occasion de discuter, m'ont eux-mêmes mis en garde contre ces chiffres. D'une part les musulmans, qui constituent le gros de la population en âge de travailler, éprouvent de la honte à se déclarer chômeurs, d'autre part plus des deux-tiers de la population sont engagés dans un processus d'émigration temporaire ou permanent. Les chrétiens constituent une communauté vieillissante de retraités, dont la descendance a pour la plupart émigré vers l'intérieur du pays, bien avant 1990.

13 Au début de la transition, la vie sociale des musulmans du village se caractérisait par un système stable et hiérarchisé de liens de parenté. Les réseaux de parenté peuvent servir d'exemple des larges imbrications de réseaux basés sur des liens de type fort (White, Houseman : 5). Dans le cas de Satovča, ils constituent un puissant cercle d'endogamie, basé sur un mariage bilatéral, monogame. Cette organisation induit un contrôle familial et de voisinage, perceptible dans la vie quotidienne, qui s'exerce sur tous les groupes d'âge selon le sexe. Dans l'espace publique, l'autorité masculine traditionnelle (islamique) est soulignée, et d'une façon générale l'autorité des plus âgés. La modernité a très lentement modifié ce schéma hiérarchique, en créant une petite cohorte de femmes éduquées, qui promeuvent une certaine émancipation économique et sociale. Même celles dont la réussite est la plus nette respectent l'autorité masculine et des personnes âgées dans l'espace publique. L'observateur étranger a l'impression que les hommes ont malgré tout l'apanage des décisions de la vie quotidienne ou à prendre en 
cas de crise; elles sont toujours prises en fonction des intérêts du lignage. Ce modèle classique que l'on peut qualifier d'islamique puise sa vitalité dans l'isolement relatif du village (et des villages faisant partie du cercle d'endogamie), dans les pressions répétées de la politique d'assimilation à l'encontre des musulmans et dans l'étroite solidarité qu'exigent des exploitations peu mécanisées, dans le cadre d'une agriculture de montagne à faible rendement.

\section{Géographie migratoire}

La grande vague migratoire pour les Bulgares musulmans de Satovča date de 1997-1998 environ. Le gouvernement socialiste de Ž. Videnov (1995-1997) fut marqué par une inflation inouïe et par la destruction du peu d'emploi qui subsistait, des acquis sociaux et d'une façon générale de toute perspective pour les habitants d'une région montagnarde frontalière. Cela confirma définitivement les musulmans locaux dans leur opinion que l'Etat bulgare menait une politique de "génocide économique »à leur encontre. L'émigration restait une solution possible.

Dans le village de Satovča, comme partout au monde, les premiers à chercher de nouvelles destinations de migrations saisonnières ou durables, furent quelques hommes qui avaient déjà une expérience de gurbet (travail saisonnier) à l'étranger. Grâce à leur expérience antérieure, ils envisageaient avec optimisme la possibilité de gagner rapidement de l'argent dans des conditions relativement correctes. Leur initiative personnelle les confrontait cependant après 1989 aux conditions nouvelles d'un marché du travail inconnu, souvent hostile et semi-légal, sans garanties ni assurances, sans traducteurs ni chefs pour prendre en charge ou répondre de leurs problèmes. Le mécanisme de base des premières migrations saisonnières sur lequel s'appuyaient ces hommes, était les liens de parenté forts. Partaient ensemble des frères, des cousins ou des beaux-frères. La décision de partir, que ce soit à court ou long terme, était prise par le cercle de parenté élargi. Les familles qui restaient au village unissaient solidairement leurs ressources (y compris financières) pour résoudre les problèmes qui survenaient en l'absence d'un membre de la maisonnée (par exemple accompagner les enfants à l'école si la mère travaille en équipe de nuit, couper et stocker le bois de chauffage pour l'hiver, etc.) Comme dans la plupart des pays en voie de développement, le travail s'est aussi féminisé en Bulgarie au cours des dernières années dans les ateliers de confection ou de chaussures; l'activité agricole s'est également féminisée (SassenKoob 1996: 1145, 1148). Les femmes ont dû prendre elles-mêmes des décisions ordinaires ou exceptionnelles, qu'elles auraient prises auparavant après consultation avec leur mari. Une partie de ces décisions ont été prises après consultation par téléphone ou par Internet avec le mari absent, une autre après avoir demandé l'avis ou l'approbation de la parenté ou des voisins. La plupart néanmoins leur incombèrent directement. En général elles ne considèrent pas ce changement comme une émancipation, mais plutôt comme une charge supplémentaire. Le souci global des femmes du village a visiblement augmenté.

16 Progressivement la géographie de la conquête du nouveau marché du travail s'est élargie. Les liens forts ont intensément fait circuler l'information, les ressources financières ont été mises en commun, pour assurer le voyage dans le pays choisi, l'épargne a commencé à arriver au village. Les défis nouveaux ont rapidement fait émerger une mythologie du succès migratoire parmi les habitants du village étroitement 
soudés. Son influence était si forte qu'en 2003 le seul fait de travailler à l'étranger était déjà devenu synonyme d'esprit d'initiative, de preuve de caractère pour les hommes, mais aussi pour les femmes, dans toutes les classes d'âge.

Au début de 2002, la commune de Satovča devint célèbre dans les média électroniques de Bulgarie pour son nombre «énorme » de migrants aux USA. Notre étude a montré qu'en fait le pourcentage d'émigrants aux USA était faible (moins de $1 \%$ ), mais la motivation pour partir était très puissante. De 1999 jusque vers 2004 presque $100 \%$ de la population adulte du village et de la commune participait à la loterie américaine pour la diversification de l'immigration (Green Card). Certains ayant gagné le droit à partir en profitèrent, d'autres y renoncèrent. Les partants se concentrèrent à Strasburg, Pennsylvania. A la base de ce choix géographique on trouve un migrant illégal d'un village voisin, qui avait réussi à gagner les USA à la fin des années 1980 . Avec la liberté de circulation, il rétablit les contacts avec sa région d'origine et se porta garant pour ses nombreux imitateurs. Ainsi ce pionnier, dont les liens au sein du réseau étaient très lâches à cause de son émigration illégale à l'époque du totalitarisme, se retrouva bientôt au centre d'un noyau nouveau. Dans ce noyau les relations de type faible (obtenir de l'information auprès d'une connaissance ou d'un parent éloigné) se sont rapidement transformées en relations de type fort: il se porte garant pour les nouveaux venus, organise les premiers temps de leur séjour sur place, sert d'intermédiaire avec l'administration locale ou les employeurs, etc. La filière de la loterie Green Card s'est très rapidement transformée en une migration en chaîne, basée sur les liens de parenté. En 2005, il y avait déjà 25 personnes du village à Strasburg, Pennsylvania, mais plus de 250 personnes de la commune, amis ou parents (cf. Karamihova $2003: 80-104$ ).

Les émigrants élaborent une topologie nouvelle de liens distendus à travers l'espace (White, Houseman : 3). Les réseaux construits sur les liens forts montrent une tendance à se constituer en cercles ou en agrégats (cluster) particulièrement nets dans l'émigration. Il en résulte une interdépendance à plusieurs niveaux, aux configurations internes complexes. Ceux qui, avant l'émigration aux USA, étaient reliés par des liens forts ou faibles constituent à présent un noyau monolithique solide; celui-ci, à distance, met en interaction les membres des réseaux initiaux en Bulgarie selon des modalités nouvelles. Le régime de transports relativement libéral, le milieu d'accueil bienveillant, qui ne fait preuve d'aucun préjugé racial ou confessionnel, l'accès à la communication et aux transports tout ceci détermine un réseau de communication très intense (presque quotidien par téléphone ou par Internet) entre les migrants et leur famille restée au village. Les vacances passées chaque année à Satovča (ou aux USA pour les membres de la famille invités) sont devenus un élément incontournable du rythme annuel. A l'automne de 2005, les habitants du village considéraient que l'attrait de l'émigration aux USA avait considérablement baissé. Il est clair que l'on a atteint la masse critique d'émigrants et que les ressources d'autres migrations à plus courte distance révèlent leurs avantages.

19 De fait, le flux à destination de l'Espagne et du Portugal, qui s'était amorcé en même temps que celui vers les USA, l'a actuellement dépassé. Le 10 avril 2004, les citoyens bulgares ont obtenu le droit de pénétrer dans les pays de l'espace-Schengen sans visa et d'y séjourner légalement pendant 90 jours (sans avoir le droit d'y travailler). L'accès facilité aux pays de l'espace-Schengen rend plus simples et plus stables les migrations de courte durée (3 mois), initiées vers 1997-1998. Simultanément les spécificités du 
marché du travail dans l'économie parallèle, mais aussi les objectifs personnellement poursuivis, exigent des séjours à l'étranger aussi longs que possible. Ainsi, ayant dépassé les trois mois autorisés, certains se sont vu interdire un nouveau séjour par un «cachet noir » sur leur passeport. Dans une telle situation, ils ont déployé tous leurs efforts pour rétablir leur droit d'accès, en faisant à nouveau jouer leurs liens forts et faibles, agissant en Bulgarie ou dans le pays concerné. Dans le flux à destination de l'Espagne et du Portugal, deux tendances se laissent clairement discerner d'ores et déjà : 1) Etablissement durable, légalisation des papiers de séjour et regroupement familial, pour les migrants les plus jeunes. 2) Navettes régulières de trois mois pour le gurbet dans l'économie parallèle: bâtiment, travail agricole, tourisme et travail domestique, pour toutes les classes d'âge. Les habitants du village affirment en plaisantant que la commune de Satovča possède déjà des filiales aux USA, en Espagne et au Portugal. C'est ainsi qu'ils caractérisent les nouveaux noyaux apparus dans leurs réseaux et qu'ils soulignent leur propre position centrale.

La plus grande demande saisonnière en travailleurs migrants semi-légaux reste la Grèce voisine. Vers le milieu des années 1990, on observe une tendance croissante chez les agriculteurs grecs les plus entreprenants à recruter des travailleurs pour la saison d'été dans les villages musulmans bulgares des Rhodopes. Ayant une bonne opinion du savoir-faire et de l'endurance de leurs voisins balkaniques, les entrepreneurs s'adressent à des couples mariés âgés de 35-40 ans. Ce travail agricole, qui dure en moyenne six mois, est relativement bien payé et est considéré, surtout par les femmes, comme un moyen de gagner de l'argent infiniment plus facile que l'agriculture domestique : après le temps de travail, elles n'ont pas le souci de la maisonnée et de l'exploitation familiale, qui accaparent leur temps quand elles sont chez elles. Durant l'été de 2005, plus de 150 personnes du village profitaient de la possibilité de travailler dans l'agriculture en Grèce. Ce travail saisonnier n'est pas vécu comme une migration. Il est perçu simplement comme un travail que l'on va chercher un peu plus loin, pour un peu plus longtemps. C'est ainsi que s'est rétablie une des destinations traditionnelles des migrations de travail saisonnier des montagnards, qui avait été coupée par les frontières politiques $\mathrm{du} \mathrm{XX}^{\mathrm{e}}$ siècle; la structure par âge et par sexe est cependant différente.

21 L'agriculture serbe permet périodiquement des migrations temporaires à de très petits groupes (des couples mariés) de Bulgares musulmans de Satovča. Les entretiens révèlent que cette destination de gurbet agricole est considérée comme peu rentable, malgré la proximité géographique et la langue slave facilitant les contacts les employeurs. Les migrations de travail vers Israël concernent de petits groupes d'hommes qui partent avec des contrats pour des entreprises de bâtiment israéliennes. Les conditions climatiques pénibles, l'insécurité permanente et des salaires en baisse expliquent l'abandon progressif de cette filière par les gurbetčii. Ils continuent pourtant à s'intéresser aux possibilités de travail là-bas et saluent volontiers en hébreu leurs invités, marquant une certaine sympathie pour le peuple israélien. Une destination tout à fait récente d'émigration (encore) temporaire a été essayée en 2005 : l'Italie, où sont partis cinq hommes pour travailler dans le bâtiment.

Jusqu'à présent les statistiques ne prennent pas en compte les habitants du village qui ont émigré durablement à l'étranger. On sait seulement avec certitude que durant l'été de 2005 cinq habitants de la commune ont été obligés de choisir entre la nationalité allemande et la nationalité bulgare. On affirme qu'ils sont « devenus allemands ». Cette 
question ne se pose pas encore pour les émigrants de longue durée dans les autres pays. Les représentants du pouvoir local ne sont eux-mêmes pas très au courant et ne s'intéressent guère à la question de la double nationalité dans la législation des pays d'accueil.

\section{Les deux extrémités des réseaux migratoires}

La majorité des partants, qu'ils soient légaux ou non, ont occupé les niches du marché du travail classiques pour des émigrants. Ce sont des emplois faiblement qualifiés, mal rétribués, en général du travail manuel pénible, sans sécurité sociale et sans accès aux avantages sociaux dont jouissent les citoyens du pays d'accueil. Leur ignorance complète de la langue locale au moment de leur arrivée et le besoin d'amasser rapidement des moyens pour s'établir, entraînent l'apprentissage laborieux et maladroit d'une sorte d'argot vernaculaire, en général au contact d'autres immigrants, d'origine slave eux aussi le plus souvent. Quelques rares individus, parmi les plus jeunes, investissent dans les études et la formation. Dans une perspective à long terme générale, cela signifie qu'au cours de leurs trois-quatre premières années, les émigrants durables s'efforcent de régler la question des papiers de séjour et d'un emploi légal dans le pays concerné. Ils travaillent pour de faibles salaires, sans cotiser à des fonds de pension privés ou sociaux.

Rares sont ceux qui parviennent à s'élever d'un cran dans l'échelle sociale après la régularisation de leur séjour. La plupart voient leur statut baisser radicalement sur l'échelle sociale, en comparaison avec celui qu'ils occupaient en Bulgarie : par exemple un instituteur devient et reste simple ouvrier du bâtiment. Ceci signifie que les salaires qu'ils touchent légalement, après un certain temps passé dans l'économie parallèle, leur assureront au mieux une retraite minimale dans le pays concerné, compte tenu de leur basse catégorie salariale et de leur temps d'activité professionnelle relativement court. A l'heure actuelle, ces retraites possibles et envisagées, qui se montent à 500-600 euros, apparaissent très satisfaisantes à l'échelle de la Bulgarie, où la retraite moyenne atteint à peine 60 euros par mois. Nombreux sont cependant ceux qui se demandent quel sera leur véritable pouvoir d'achat après l'admission tant attendu de la Bulgarie dans l'Union Européenne (janvier 2007) et le probable rattrapage des salaires et des prix par rapport à l'Europe. Une des réponses relevées dans nos entretiens à Satovča a été : «Ils en reviendront au tabac sur leurs vieux jours, tout comme nous! » Leurs velléités d'occuper des positions plus élevées dans la hiérarchie villageoise grâce à leurs revenus actuels apparaissent donc vouées à l'échec à long terme. Le fait de résider dans un milieu étranger, que l'on peut supposer plus ouvert à la modernité et à la globalisation, ne leur vaut pas un prestige social supplémentaire, du fait de la perception très nette de leur statut social dégradé «là-bas". Les visites de travail qu'effectuent les villageois auprès de leurs proches en émigration ont largement contribué à une meilleure connaissance des conditions de vie réelles à l'étranger. En 2005, j'ai pu observer l'érosion de pans entiers de l'idéologie migratoire, que j'avais relevés en 2002. Il m'a semblé que les démonstrations ostentatoires d'un niveau de vie élevé de la part des émigrés, ce qui était une des composantes majeures de cette idéologie, sont actuellement à contre-courant de la sensibilité migratoire; elles sont considérées comme des dépenses inutiles et sont désapprouvées. 

village natal. J'ai noté en 2002 le premier achat de maison par une jeune famille émigrée aux USA. La mythologie migratoire locale affirmait que cette famille était particulièrement pauvre, mais qu'en deux années d'émigration elle avait mis de côté suffisamment d'argent, non seulement pour venir en vacances, mais même pour acheter la maison des douaniers. En 2005, le marché immobilier était particulièrement animé à Satovča. On m'a signalé et ont m'a présenté les cas d'un certain nombre d'émigrants "très pauvres ", qui, ou bien avaient acheté une maison au bout de deux ou trois ans à l'étranger, ou bien retapaient de fond en comble leurs maisons familiales. Ces investissements sont indicatifs d'une part des stratégies familiales visant à s'assurer d'un logement de qualité pour les années de retraite, que l'on prévoit de vivre en Bulgarie, dans le noyau même du réseau. D'autre part, on peut l'interpréter comme un investissement immobilier, qui pourra être avantageusement revendu plus tard. Depuis trois ans environ, en effet, dans différents coins du pays, des maisons sont achetées par des étrangers venant littéralement de tous les coins de la planète (Japonais, Anglais, Albanais, etc.) ce qui a fait flamber les prix de l'immobilier. Pour la première fois en Bulgarie, les gens commencent à considérer la maison comme une marchandise, et non plus comme un nid familial lourd d'une charge sentimentale et sociale. Le paradoxe, c'est que dans ces success stories apparaissent des familles nucléaires, coupées de leurs réseaux familiaux ou autres, qui, toutes seules («au prix d'un travail honorable») parviennent à la réussite. Ces premières indications de la mise en avant de l'individu (néanmoins inclus dans un couple marié) par rapport au groupe, donc en dehors de tout réseau, marquent une tendance très intéressante.

La distance sociale sur laquelle s'échelonnent les membres du groupe est encore faible. Cela tient d'une part à la stratégie efficace de nivellement social et économique pratiquée par le socialisme. D'autre part, le statut relativement inférieur des émigrés sur le marché du travail et les dépenses importantes qui leurs sont imposées, ne permettent pas l'ouverture rapide de "ciseaux » sur le plan social entre ceux qui sont restés au pays et les migrants. Quoique les revenus financiers des familles de Satovča soient modestes, grâce à la mobilisation des liens forts, ils peuvent assurer, par le seul investissement du travail, leur nourriture tout au long de l'année (selon certaines sources c'est le poste budgétaire le plus important des ménages en Bulgarie : jusqu'à 45 $\%)$, ils habitent des maisons dont ils sont propriétaires, qui ont été assez bien équipées durant la période précédente, ils utilisent l'important trousseau traditionnel des épouses (vêtements et tissages). La grande solidarité locale, combinée avec le modèle éthique balkanique de responsabilité partagée envers les gens dans le besoin, renforcé d'ailleurs par la conception musulmane de la charité, rend la redistribution de tous les biens à l'intérieur du groupe obligatoire et interdit d'admettre une pauvreté extrême. Tout ceci explique la faible distance sociale entre les différents petits noyaux du réseau (White, Houseman : 3). Ce mécanisme connaît une très lente érosion, en raison des facteurs énumérés précédemment.

La solidarité économique est un élément-clé du fonctionnement de réseaux tels que ceux que nous étudions. Les ressources sont redistribuées par les économiquement forts vers ceux qui sont dans le besoin. Bien qu'en général plutôt mal payés, les émigrants gagnent et économisent davantage d'argent que leurs proches à Satovča, en particulier que les retraités âgés. En 2002, j'entendais sans cesse parler d'aide financière attendue par les proches des émigrés. Les parents âgés la mentionnaient souvent 
comme quelque chose qui leur était dû. En 2005, ce thème n'apparaît plus dans les conversations, à l'exception du cas d'une femme très malade qui reçoit périodiquement de petites sommes de son fils travaillant illégalement en Espagne. Assurément les émigrés continuent d'envoyer de petites sommes selon la voie traditionnelle (par l'intermédiaire de quelqu'un qui fait le voyage) à leurs parents âgés et en cas d'urgence à des membres de la famille au sens large. Mais l'attente de base de cette solidarité serait à présent plutôt l'aide fournie au nouveau partant par ses proches et connaissances pour s'installer facilement lors de ses débuts à l'étranger. Il semble que l'on soit parvenu à un équilibre entre "ici » et "là-bas » et l'aide financière est désormais répartie là où le besoin s'en fait sentir, ce qui n'est plus nécessairement à Satovča même.

Mes observations de 2005 m'ont permis de constater que les habitants de Satovča ont élaboré un modèle propre de stratégie de réussite à court ou à long terme. Ce modèle n'exclut pas les séjours de gurbet à court terme en Europe. Mais en 2005, il n'est plus suggéré qu'il faille émigrer "à tout prix ", ce qui était martelé trois ans plus tôt. Les habitants de Satovča semblent pour la première fois émancipés sur le plan économique et avoir pris quelques distances avec leurs co-villageois partis en émigration. Une distance commence à se marquer entre le noyau initial du réseau, localisé au village natal, et les nouveaux agrégats (cluster) formés par les émigrants de longue durée en Europe et en Amérique. Il est évident que les modifications des circonstances induisent des modifications des réseaux. De nouveaux réseaux viennent s'imbriquer : ceux des émigrés entre eux et leur progressive ouverture vers les habitants du pays d'accueil (pour l'instant des liens de type faible), mais aussi ceux des restés au village entre eux (avec des liens faibles qui prennent de l'importance). Les différents réseaux, localisés différemment, se recouvrent pour l'instant dans la plupart des domaines et constituent un ensemble commun distendu dans l'espace, à l'intérieur duquel les liens individuels, de type fort ou de type faible, ne cessent de se transformer. On observe parallèlement un cycle de globalisation et de renforcement des réseaux régionaux, aussi bien à Satovča que dans les agrégats (cluster).

Pour l'instant les ressources du marché du mariage dans la commune, avec laquelle coïncide le cercle endogamique, sont suffisantes. Dans la stratégie encore hésitante des deux groupes, celui des migrants définitifs et celui des migrants temporaires, on n'observe pas de tendance à une ouverture rapide du cercle de mariage (avec des femmes ou des hommes du pays d'accueil) comme élément possible d'une intégration mieux réussie dans les sociétés locales. Pour l'instant, à l'instar de toutes les descriptions d'émigrants du XX siècle, on « importe » des femmes de son propre cercle endogamique.

\section{Politique et religion}

L'accès au pouvoir, aux centres de décision et d'attribution de ressources au niveau local, régional ou national est un facteur majeur pour le succès des stratégies de réussite collectives élaborées et entretenues par les différents réseaux: ce sont les représentations politiques qui s'en chargent. En Bulgarie, le principal représentant politique des musulmans est le Dviženie za Prava i Svobodi (DPS : Mouvement des Droits et Libertés), créé le 26 mars 1990 à Sofia et dont le leader est Ahmed Dogan. C'est un secret de polichinelle qui revient périodiquement dans le débat publique, que, en 
contradiction avec la Constitution, le DPS est de facto un parti ethnique qui représente les Turcs de Bulgarie. Dans les Rhodopes occidentaux, le soutien des Bulgares musulmans au DPS est très puissant. Les Bulgares musulmans de Satovča votent massivement pour ses représentants. Ils estiment que cela les protège d'une nouvelle politique d'assimilation. Leur accès réel au pouvoir et aux ressources, par ce biais, reste limité au niveau du pouvoir local. Ils n'occupent de leadership dans le DPS qu'à un niveau local (Marushiakova \& Popov). Si bien que le noyau de pouvoir et de contrôle qui crée et exige l'existence d'un réseau fonctionne dans un cadre géographique relativement étroit. Au niveau local, il se confond avec les quelques lignages qui occupent traditionnellement des positions dominantes. Les liens de type fort consolident ainsi leur existence dans le nouveau contexte politique aussi. Les faibles ressources de l'économie locale et par conséquent l'intérêt médiocre qu'il y a «à être au pouvoir » (ou tout au moins à en être proche) sont tels qu'on pourrait s'attendre à ce que ces liens se déploient à un niveau d'importance assez faible. Il est important de noter, cependant, que l'action du DPS sert aussi à construire et à stabiliser l'identité musulmane, tant au niveau national que régional.

31 L'opinion générale en Bulgarie associe traditionnellement l'islam à des membres de la société pauvres et sans éducation. Longtemps l'islam était l'attribut visible des personnes les plus âgées, de ceux qui à la fin de leur vie espéraient se purifier de leurs péchés et s'assurer une garantie de survie dans l'au-delà. Je constate qu'au début du $\mathrm{XXI}^{\mathrm{e}}$ siècle cette image commence à se modifier : à Satovča les porteurs de l'islam sont désormais de jeunes intellectuels, ayant une éducation à la fois laïque et religieuse, exerçant une forte influence (y compris sur le plan économique) sur le milieu local et jouissant d'appuis politiques solides. A la fin des années 1990, le village et la commune avaient envoyé quelques jeunes gens faire des études dans des universités islamiques d'Arabie Saoudite et de Jordanie. En 2002, je n'avais rencontré qu'un seul garçon, en sixième classe du primaire, qui étudiait systématiquement le Coran et souhaitait expressément poursuivre ses études secondaires à l'école confessionnelle de Momčilgrad, dans la partie orientale des Rhodopes. Son père était le premier à formuler clairement le discours, qui en 2005 est visiblement devenu une norme admise, selon lequel le retour à l'islam garantit le succès en affaire. Dans ce cas concret, le père avait cessé de vendre de l'alcool dans son petit magasin et affirmait qu'Allah l'avait récompensé dans son commerce. En 2003-2004 de jeunes diplômés en sciences religieuses islamiques, dont personne ne parlait en 2002, sont revenus et occupent des fonctions dans les mosquées et les écoles du village et de la commune.

Dès 2005, les paramètres des changements en profondeur que les jeunes voulaient promouvoir étaient perceptibles. Leur objectif était de changer fondamentalement aussi bien la vie intérieure, intime des musulmans, que d'affirmer la présence extérieure, publique, de la religion "nouvelle et pure ». Pour l'instant les "savants", ainsi qu'on nomme les réformateurs dans le village, luttent pour modifier trois pratiques importantes dans la vie religieuse de la communauté. Toutes trois interviennent dans le cycle rituel le plus dramatique: celui des funérailles et des commémorations funéraires (mevlid) qui touchent l'essence la plus intime de la culture locale. Cette tentative de modifier radicalement le domaine le plus fondamental et profondément intime de la vision du monde, les rituels de passage, suscite de vives réactions. Bien que partant de positions radicales, les jeunes s'efforcent de comprendre leurs opposants plus âgés. « Les vieux ont l'habitude de tenir pour bon ce qu'ils ont fait 
depuis 30 ou 40 ans " admettent-ils. "Etions-nous donc sur la mauvaise route depuis tant d'années ?! » ripostent leurs aînés. A l'heure actuelle, la tendance observée ne semble pas mener à un conflit ouvert. Les plus âgés s'en sortent plus simplement en ayant recours à de « vieux » hodjas, venus de l'extérieur. Simultanément cependant, ces personnes atteintes dans leur conviction la plus intime, recourent au discours commode, servi par les médias nationalistes selon lequel l'islam moderne est par définition lié au fondamentalisme et au sectarisme. Les jeunes sont étiquetés «talibans » dans les cancans villageois. Et pour être sûrs que les usages seront respectés, les vieux recourent à la croyance, toujours très vivace, à la fonction performative de la parole: «On dit ici : si vous ne me faites pas de mevljud, je vous maudirai depuis la tombe!»

Les villageois ne surmonteront évidemment pas ce clivage interne à la communauté tant que la génération ancienne est en vie et tant que les non-pratiquants s'en tiennent à leur mode de vie moderne et confortable, affranchi des règles restreignant la vie quotidienne dans un Etat laïc, mais par définition chrétien orthodoxe. Les «savants " de leur côté sont fortement motivés pour introduire des réformes. Ils ont adopté une stratégie efficace en agissant sur la génération la plus malléable : les jeunes écoliers. L'idée dominante de cette réforme est l'édification d'un système de valeur et d'un modèle comportemental vigoureux, clairement défini comme musulman. Durant l'année scolaire 2001-2002, pour la première fois en Bulgarie, on a introduit dans les régions mélangées un enseignement religieux obligatoire, avec une option musulmane, financée par le Bureau du Grand Mufti (Glavno Mjuftijstvo). En août 2003, le Grand Mufti Selim Mehmed a présenté les premiers manuels de religion pour les écoles (de la première à la huitième classe). Ils sont gratuits et ont reçu l'approbation du Glavno Mjuftijstvo et du Ministère de l'Education et des Sciences. A Satovča la nouvelle matière a été immédiatement introduite. A l'heure actuelle il y a cinq groupes pour les $1^{\text {ère }} 4^{\text {ième }}$ années et neuf pour les $5^{\text {ième }}-12^{\text {ième }}$ années, ce qui rassemble $40 \%$ des élèves. Pour les enfants des quatre premières classes, ce sont les mères qui décident, c'est pourquoi certains d'entre eux n'y participent pas. L'année dernière « ...a faiblement marché dans le cours supérieur, sauf si les parents sont impliqués dans l'islam, s'ils vont à la prière du vendredi et s'ils font les cinq namaz (prières).» L'intérêt nouveau qui s'est créé pousse les enfants à chercher une information complémentaire. Les écoliers regardent la série télévisé brésilienne "Cloning", dans le genre soap opera, qui présente la vie de riches musulmans. Dans les entretiens sur le thème de la religion avec les habitants de Satovča, les deux mots clés étaient «les savants» et "la connaissance». Ainsi les transformations en cours sont-elles présentées sous une étiquette moderne, comme un mouvement progressiste au niveau des idées et de la pratique.

On considère traditionnellement que ce sont les hommes qui pratiquent l'islam et le représentent dans l'espace publique. Dans une situation où les hommes en âge de travailler sont perpétuellement absents, un nouveau processus de prise de décision s'esquisse où s'affirme l'influence du monde féminin. En 2005, les hodjas «savants » ont reçu le ferme soutien de femmes jeunes ayant un emploi, dont les interviews montrent qu'elles se sont mises à s'intéresser à la théorie et à la pratique de l'islam, introduisant peu à peu dans leur foyer le jeûne du ramadan (vers 2004). Indubitablement les femmes sont les "gardiennes des liens de parenté " qu'elles entretiennent et utilisent plus souvent que les hommes (Wetherell, Plakans, Wellman, 1994 : 649). Ainsi, encouragées 
par les jeunes hodjas, les femmes font appel aux ressources des réseaux de type fort ou faible pour introduire de nouvelles normes comportementales dans le village.

La seconde entreprise d'importance à laquelle se consacrent actuellement les " jeunes » est la construction d'une mosquée de taille imposante dans le village. Le comité d'initiative a élaboré un projet et cherche des financements, aussi bien auprès de fondations musulmanes que des locaux et des émigrés. Cette initiative est accueillie avec des sentiments mitigés. Une partie des habitants ne croit pas que l'on parviendra à rassembler les fonds nécessaires. Ils redoutent que l'on ne détruise l'ancienne mosquée datant des années 1930 , et que l'on reste ensuite sans lieu de prière. D'autres s'inquiètent de faire à nouveau l'objet de critiques de la part de la majorité chrétienne, car « la nouvelle mosquée ne fera qu'agacer les Bulgares».

La hiérarchisation interne des réseaux à Satovča comme dans l'émigration est sans nul doute en pleine évolution. Le principe de base de la hiérarchie est en train de se modifier : le poids des jeunes (les enfants des émigrés, les enfants ou le frère cadet resté au village) augmente au point de prendre des décisions de plus en plus importantes, car ils peuvent s'adapter plus vite aux conditions nouvelles. La tendance à une légère modification de la position de la femme (la fille, l'épouse) prend des dimensions nouvelles, du fait de l'adoption plus massive du modèle comportemental islamique publique de la part des jeunes du village. Comment cette « islamisation» réformiste du noyau de base se répercute-t-elle sur les relations avec les nombreux émigrés temporaires ou durables?

Une bonne partie du dialogue entre les « locaux » et les émigrés se formulait en 2005 en ces termes: "On réussit, aussi bien ici qu'en émigration, si on est un musulman croyant!» Les leaders spirituels locaux, avec une bonne dose de fatuité, commentent l'éloignement des émigrés par rapport à la foi. Ils disent que « toute la foi de la plupart des émigrés se résume au fait de porter des amulettes (muska) ». «Porter des muskas n'a de sens que si on croit que c'est la parole d'Allah qui y est inscrite et qu'on a fortement la foi. Quand ils partent pour l'étranger, ils vont voir le hodja : «Hodja, écris-moi une muska pour que mon voyage se passe bien et que je sois bien là-bas!» Ils s'écartent de la foi, ils n'ont d'attention que pour la muska, qu'ils suspendent au rétroviseur de leur voiture pour qu'elle y pendouille. Le croyant n'a pas besoin de muska, il s'adresse directement à Allah pour avoir Sa protection. Celui qui n'a pas la foi peut bien avoir cent muskas...»

Nous observons donc un processus déjà bien avancé de stabilisation du grand noyau local des réseaux, sur le thème de la religion, ainsi qu'un éloignement croissant, qui n'est plus seulement géographique, d'avec les communautés émigrées. C'est sur ce thème aussi que les liens forts se relâchent. Ce processus est favorisé par différents facteurs: l'Etat, par l'intermédiaire du Ministère de l'Education et des Sciences, encourage le développement de l'éducation musulmane. Après avoir accepté que la matière Religion soit étudiée dans toutes les classes, le ministère ne contrôle pas le contenu du processus éducatif. De facto, le système éducatif laïque bulgare laisse le champ libre à la propagande et la formation religieuses dans les écoles d'Etat. Le Bureau central du mufti à Sofia fournit gratuitement des manuels à tous ceux qui en font la demande. Ils ont été écrits par des hommes de religion arabes et véhiculent les discours caractéristiques des différentes tendances spirituelles auxquelles appartiennent leurs auteurs. Le Glavno Mjuftijstvo organise des cours pour les enseignants musulmans ayant démontré leur attachement à la religion. Il assure aussi 
les moyens financiers permettant aux enseignants (y compris ceux de Satovča) de se rendre dans la monde arabe. Diverses universités et fondations islamiques en Arabie Saoudite et en Jordanie assurent une formation pédagogique supérieure aux musulmans, financent des écoles religieuses privées et contrôlent directement l'éducation dans les écoles musulmanes d'Etat. Les communautés locales, qui ont subi les tentatives d'assimilation répétées de l'Etat bulgare tout au long du $\mathrm{XX}^{\mathrm{e}}$ siècle, en réponse à cette violence passée, encouragent ces transformations par le biais de leurs structures familiales, de parenté, de voisinage et par leurs réseaux de groupes.

Certains facteurs nuancent pourtant la rapidité et l'ampleur du processus. Tous les groupes ethno-culturels de Bulgarie, et parmi eux les Bulgares musulmans, avaient atteint un fort degré de sécularisation à la fin du $\mathrm{XX}^{\mathrm{e}}$ siècle. Construire une vision du monde profondément religieuse est un objectif difficile et lent à atteindre à notre époque laïcisée. Les tentatives d'assimilation de la monarchie bulgare (1878-1944) et de l'Etat totalitaire (1944-1989) ont créé un sentiment d'insécurité (la crainte de brusques revirements) qui structure le quotidien des Bulgares musulmans. Elles entretiennent la conviction d'une grande partie des villageois qu'il convient de se donner les apparences de citoyens loyaux, qui ne font pas étalage de leur appartenance religieuse, afin de ne pas susciter de nouvelles mesures d'assimilation de la part du pouvoir. Les stratégies migratoires dans le monde contemporain, particulièrement après le 11 septembre 2001, imposent un mimétisme identitaire aux émigrants. L'émigration, en bonne partie illégale, vers les pays chrétiens impose d'abandonner l'identité musulmane, ou tout au moins de la "dissimuler " à l'intérieur de la maison. Dans l'espace publique, on ne manifeste pas les signes identitaires extérieurs; les noms officiels des émigrants n'ont pour la plupart pas de consonance musulmane. Le modèle de pratique religieuse qui a largement cours en Bulgarie est lié aux personnes âgées à la retraite; il libère psychologiquement pour l'instant les émigrants d'avoir à pratiquer leur religion. Ils travaillent avec acharnement à l'accumulation initiale de moyens matériels et reportent à plus tard l'adoption des pratiques religieuses « pour quand ils vieilliront et reviendront au pays. »

40 La plupart des émigrés se retrouvent avec un statut social infériorisé, des liens affaiblis avec leurs réseaux habituels (y compris la parenté), une identité instable, fluctuante, en cours d'adaptation au pays d'accueil. Simultanément, la plupart de ceux qui sont restés au village voient leurs revenus stabilisés, utilisent la nouvelle politique de crédit bancaire, développent avec succès une stratégie durable de développement pour leur famille et leurs enfants, entretiennent et élargissent des réseaux sociaux stables, intègrent progressivement les mutations de leur société, sans ruptures, et adoptent peu à peu de façon durable la «nouvelle » identité musulmane porteuse de prestige.

41 Ainsi, le noyau du grand réseau local qui envoie ses migrants au loin cesse d'occuper une position passive. Il se met à exercer une influence sur les migrants selon divers mécanismes, y compris la prochaine adhésion de la Bulgarie à l'UE, qui transforme le village natal (le point nodal du réseau) en un lieu attractif, où les émigrés peuvent investir, à l'heure actuelle principalement par l'achat de maisons luxueuses. Les habitants locaux préparent activement ce changement, ils sont bien insérés dans les structures locales, ils protègent systématiquement leur cadre de vie. Ils mettent la barre haut dans la compétition pour l'étalage de leur niveau de vie et de leur statut social qui ne cesse de s'élever (business prospère, revenus du tabac, éducation supérieure pour les enfants). Dans cette compétition, le seul fait de gagner de l'argent 
et de déployer du « luxe à crédit » de la part des migrants a déjà beaucoup perdu de son prestige. Les réformateurs musulmans proposent un ensemble de valeurs dont la nécessité se fait dramatiquement ressentir pour chaque habitant, dans un monde aussi plein de tragédies et de conflits, dominé par les médias et globalisé que le nôtre. Cet ensemble de valeurs a l'avantage de se présenter auréolé par la tradition, mais aussi rénové par les processus de modernisation en cours dans le monde musulman. L'islam sous sa forme réformée propose une identité clairement rénovée au groupe et à chacun des individus qui le compose. Ses propagandistes sont des habitants locaux, prospères et jouissant d'autorité, issus de familles respectables. La variante modernisée de l'islam propose une identification nouvelle, élargie au vaste réseau de l'Umma, laquelle associe pour la première fois la population locale, frontalière, d'une région plutôt pauvre et considérée comme arriérée, avec le monde fastueux des cheikhs du pétrole auquel est confié la tâche d'assurer la garde des Lieux Saints au nom des croyants.

\section{Conclusion}

Nous sommes donc témoins d'un nouveau processus en cours. Le milieu initial dont émanent les migrants ne se trouve désormais plus en situation passive. Satovča n'attend pas de la part de ses émigrés de l'argent, des technologies nouvelles, des connaissances, des valeurs, un style de vie, comme c'était le cas pour les régions de migrations classiques. Au contraire, nous voyons les leaders du noyau central du réseau adopter une position active nouvelle, qui exerce son action sur les migrants, leur pose des conditions et leur impose certaines valeurs et normes comportementales nouvelles. L'adoption et le déploiement ostentatoire de l'identité musulmane deviennent une des conditions pour l'émigration temporaire, afin de maintenir facilement et sans conflits les liens de type fort. Une partie des migrants a déjà adopté les nouvelles règles. Je suis convaincue que les émigrés ayant le mieux réussi investiront le plus dans la construction de la nouvelle mosquée, car c'est un comportement migratoire classique, quelle que soit de la religion pratiquée. Ce sera pour eux un moyen d'affirmer leur statut prééminent au sein de la communauté et de valider la justesse de leur choix d'émigrer. En fait, ils se soumettront aux normes dictées par le milieu natal où se concentre la vitalité du réseau auquel ils appartiennent. Nous pouvons cartographier la dynamique des nœuds du réseau selon une logique centre-périphérie. Au départ les émigrants, indépendamment de l'endroit où ils se sont établis, ont eu tendance à s'affirmer comme centre de gravité ; en 2005, Satovča rétablit clairement sa position de centre le plus actif, par le biais des nouvelle tendances religieuses.

La situation marginale du groupe des Bulgares musulmans sur une période historique suffisamment longue, combinée avec la situation d'isolement relatif du village liée au contexte montagnard frontalier et à l'économie à dominante agricole, confèrent une importance exceptionnelle aux réseaux familiaux et de proximité communautaire, avec un recours intensif aux liens de type fort (durables). Les processus migratoires entretiennent ces liens très solidement et n'incluent qu'un nombre relativement faible de liens de type faible (à court terme), servant fondamentalement à résoudre des problèmes concrets majeurs. Ce vaste réseau qui englobe de petits noyaux éloignés géographiquement continue d'être dominé par son centre actif, le village de Satovča, qui renforce ses positions en s'inscrivant activement dans les réseaux plus vastes du monde musulman, et quoiqu'à un moindre degré, dans ce qu'il perçoit comme sa 
propre représentation politique. La différenciation sociale encore très faible maintient les liens de type fort à l'intérieur des noyaux et entre eux, ainsi qu'entre les individus qui les composent. Je considère que les processus liés à l'édification d'une identité claire (islamique ou autre) seront des facteurs décisifs pour le maintien ou pour la lente désagrégation du réseau au sens large.

\section{BIBLIOGRAPHIE}

Alexandrov, H. (1998) Breznitsa : Social Assessment Study. Report. World Bank, September 1998. Une variante de ce rapport a été publiée en bulgare : Aleksandrov, H. (2000) Rodsvoto, način na upotreba v edno selo. Antropologični izsledvanija, T.1. NBU, 161-178.

Bjuksenšjuti, U. (2000). Malcinstvenata politika v Bălgarija. Politikata na BKP kăm evrei, romi, pomaci i turci.(La politique de la Bulgarie envers les minorités. La politique du PCB envers les juifs, les Roms, les Pomaks et les Turcs) Izd. MCPMKV.

Bougarel, X. Islam and Politics in the Post-Communist Balkans http://www.ksg.harvard.edu/ kokkalis/GSW1/GSW1/13\%20Bougarel.pdf

Georgieva, Ts. \& Zhelyazkova, A. (1994) L'identité en période de changement (Observations sur certaines tendances du monde mixte des Rhodopes), Cahiers Internationaux de Sociologie, vol. XCVI, pp. 125-143.

Ghodsee, K. (2005) Research Report http://www.irex.org/programs/iaro/research/05-06/ Ghodsee.pdf.

Gruev, M.(2003) Meždu petolăčkata I polumeseca. Bălgarite mjusjulmani I političeskijat režim (1944-1959) (Entre l'étoile à cinq branches et le croissant. Les Bulgares musulmans et le régime 1944-1959). IK "KOTA".

Dajnov, E. (2002) Za kakvi gi mislim ? Obrazite na malcinstveni i specifični obštnosti v Bălgarija, razkriti v naučni izsledvanija. (Que pensons-nous d'eux ? Les figures des communautés minoritaires et spécifiques en Bulgarie, à la lumière des études scientifiques) Tehnite glasove. Prisăstvieto na malcinstveni I specifični obštnosti v bălgarskite medii (kraja na 20-ti -načaloto na 21 vek) (Leurs voix. La présence des communautés minoritaires et spécifiques dans les médias bulgares (fin du XX ${ }^{\mathrm{e}}$ et début du XXI ${ }^{\mathrm{e}}$ siècle)), CSP, 13-30.

Ethnobarometer http://www.ethnobarometer.org/pagine/Bulgaria\%20 paper.pdf.

Željazkova, A., V. Grigorov, D. Dimitrova (2005) Imigranti ot Blizkija i Srednija iztok (les immigrants du Proche et du Moyen Orient). Imigracijata $v$ Bălgarija (L'immigration en Bulgarie) (ed. A. Krăsteva), MCPMKV, 19-73.

International Migrations from Countries with Economies in Transition : 1980-1999. population Division, DESA, UN Secretariat 11.09.2002.25.

Ivanov, A. (1998) Minority Nationalism in the Balkans : The Bulgarian Case. http://ime-bg.org/ pdf_docs/papers/minority.pdf. 
Karagiannis, E. An Introduction to the Pomak Issue in Bulgaria. http://www.ethno.unizh.ch/ mitarbeiterinnen/Introduction_Pomak_Issue_Bulgaria.pdf.

Karamihova, M.(1999) T. nar. "văzroditelen proces “ politika i rezultati (Le prétendu « Processus de Régénération", politique et résultats). Za promenite... Izd. CSP

Karamihova M. (2003) Emigracijata ot Rodopite, nov fenomen ili vremenen otgovor v perioda na kriza? (L'émigration des Rhodopes, un phénomène nouveau ou une réponse temporaire en période de crise ?). Da živeeš tam, da se sănuvaš tuk. Emigracionni procesi v načaloto na XXI vek (Vivre ici, se rêver là-bas. Processus migratoires au début du XXI ${ }^{\mathrm{e}}$ siècle), (ed. Karamihova). Izd.

MCPMKV, 23-104

Konstantinov, Yu. (1997) Strategies for Sustaining a Vulnerable Identity : The Case of the Bulgarian Pomaks, Muslim Identity and the Balkan State, (eds. H. Poulton \& S. Taji-Farouki), London, Hurst and Co, pp. 33-53.

Marushiakova, E. \& Popov, V. The Muslim Minorities in Bulgaria http//www.emz-berlin.de/ projektepj41_1.htm

Mihail, D. (2003) Ot "mestna" kăm "evropejska" identičnost : promenjašite se identičnosti na pomaškoto malcinstvo v Gărcija (D’une identité "locale" à une identité "européenne" : changements identitaires de la minorité pomaque de Grèce). Da živeeš tam, da se sănuvaš tuk. Emigracionni procesi v načaloto na XXI vek (Vivre ici, se rêver là-bas. Processus migratoires au début du XXI ${ }^{\mathrm{e}}$ siècle), (ed. Karamihova). Izd. MCPMKV, 248-266

Neuburger, M. (1997) "Difference Unveiled : Bulgarian National Imperatives and the Re-Dressing of Muslim Women in the Communist Period 1945-1989". Nationality Papers, n¹, pp. 169-181

O'Learly, B. McGarry, J. (eds) (1993) The Politics of Ethnic Conflict Regulation: Case Studies of Protracted Ethnic Conflicts. London Routledge.

Sassen-Koob, S. (1996) Notes on the Incorporation of Third World Women into Wage-Labor trough Immigration and Off-Shore Production, The Sociology of Migration (ed. R. Cohen), Vermont.

Pickles, J. (2001) "There are no Turks in Bulgaria" : Violence, Ethnicity and Economic Practice in the Border Regions and Muslim Communities of Post-Socialist Bulgaria. Max Planck Institute for Social Anthropology, Working Papers $n^{\circ} 25$.

Stojanov, V. (1998) Turskoto naselenie v Bălgarija meždu poljusite na etničeskata politika (La population turque de Bulgarie entre les pôles de lapolitique ethnique).

Todorova, M. (1997) “Identity (Trans)Formation among Pomaks in Bulgaria”. Beyond Borders. Remaking Cultural Identities in the New East and Central Europe (eds. L. Kürti \& J. Langman) Westview Press, Boulder, pp. 63-82

White, D. \& Houseman, M. The Navigability of Strong Ties : Small Worlds, Tie Strength and Network Topology. http://www.santafe.edu/sfi/publications/Working-Papers/02-10-055.pdf.

Wetherell, Ch. A. Plakans, B. Wellman (1994) Social Networks, Kinship and Community in Eastern Europe. Journal of Interdisciplinary History, XXIV : 4 (Spring), 639-663

\section{RÉSUMÉS}

L'article propose une analyse du jeu des réseaux sociaux dans un petit village bulgare de montagne, situé à la frontière avec la Grèce et confronté aujourd'hui à d'intenses mouvements migratoires. Les habitants de Satovča sont des Bulgares musulmans; ils appartiennent à un 
groupe qui a été soumis par l'Etat bulgare à une politique d'assimilation pendant tout le XXème siècle. Sont étudiés les liens hétérogènes à travers lesquels s'établissent des réseaux sociaux dynamiques et entremêlés de façon complexe. La centralité des réseaux locaux - sociaux comme de parentèles - au sein desquels des liens forts et durables sont mobilisés s'explique par la conjugaison entre la situation sociale marginale des Bulgares musulmans sur la longue durée et le relatif isolement du village étudié (en raison de sa localisation périphérique et montagneuse et de son économie avant tout agricole). Les processus migratoires contemporains confèrent à ces liens un grande stabilité dans la mesure où les mobilités ne font intervenir qu'un nombre très limité de liens faibles (de courte durée) principalement consacrés à la réalisation de tâches concrètes. Le large réseau qui comprend de plus petits noyaux géographiquement éloignés est dominé par le village de Satovča qui puise des ressources complémentaires dans son insertion active dans le monde musulman plus vaste ainsi que dans la participation, même limitée, à une représentation politique vécue comme propre. La différenciation sociale encore minimale à l'intérieur des groupes de Bulgares musulmans rend possible l'entretien de liens à l'intérieur du noyau nodal comme entre ce dernier et les individus qui le constituent. On peut avancer à titre d'hypothèse l'idée selon laquelle le travail de définition d'une identité claire, qu'elle soit musulmane ou autre, constituera un facteur décisif pour l'avenir de ces réseaux, leur préservation ou leur délitement progressif.

\section{INDEX}

Index géographique : Bulgarie, Rhodopes

Mots-clés : Migrations, Réseaux sociaux, Musulmans

\section{AUTEURS}

\section{MARGARITA KARAMIHOVA}

Dr. Margarita Karamihova est diplômée d'histoire et d'ethnologie. En 1991 elle a soutenu une thèse de doctorat sur « Le mariage sur les terres bulgares pendant la période de domination ottomane ». Directeur de recherche à la Section d'ethnologie balkanique de l'Institut et du Musée d'ethnographie de l'Académie des sciences bulgares (BAN) et enseignante à l'Université de Sofia « Kliment Ohridski » ainsi qu'à l'Académie nationale de police, elle travaille sur les dynamiques culturelles chez les musulmans et les groupes ethnoculturels qui les entourent dans les Balkans avec, pour principaux champs de recherche, la famille, le pèlerinage, les migrations et les politiques publiques (notamment socialistes) des minorités. Elle a récemment publié : «Identities Palette in the Course of European Integration : A Case Study of Satovcha Village », in : Elena Marushiakova (ed.), Dynamics of National Identity and Transnational Identities in the Process of European Integration, Cambridge : Cambridge Scholars Publishing, 2008, p.248-270 ; « Les Bulgares musulmans dans la vie politique après 1989 : un groupe politique anonyme ", Revue d'études comparatives Est - Ouest, 38(4), déc. 2007, p.29-48 ; « C'est un signe du destin! La dynamique des rapports entre un groupe d'émigrés et leur pays natal », In : Vivre ensemble au XXème siècle ? Actes du colloque international de l'Institut de sociologie de l'Université libre de Bruxelles, Bruxelles : ULB, 2007, p.427-446; Gradivo za etnologija na migraciite [Contribution à l'ethnologie des migrations] (sous la dir.), Sofia : EIM, 2006.

mkaramihova_m@abv.bg 